\title{
Investigations for a Type of Variable Coefficient Fractional Subdiffusion Equation with Multidelay
}

\author{
Wei Gu \\ School of Statistics and Mathematics, Zhongnan University of Economics and Law, Wuhan 430073, China \\ Correspondence should be addressed to Wei Gu; wei_gu@zuel.edu.cn
}

Received 3 July 2020; Accepted 27 July 2020; Published 11 August 2020

Academic Editor: Liguang Wang

Copyright (c) 2020 Wei Gu. This is an open access article distributed under the Creative Commons Attribution License, which permits unrestricted use, distribution, and reproduction in any medium, provided the original work is properly cited.

A difference scheme is constructed for a type of variable coefficient time fractional subdiffusion equation with multidelay. Stability and convergence results of the scheme are obtained, and theoretical results are proved by two numerical tests.

\section{Introduction}

This paper will consider the following variable coefficient time fractional subdiffusion equation with arbitrary multidelay

$$
\begin{aligned}
\frac{\partial^{\alpha} u}{\partial t^{\alpha}}-\left(D(x) u_{x}\right)_{x}= & f\left(u(x, t), u\left(x, t-s_{1}\right), u\left(x, t-s_{q}\right), x, t\right), \\
& (x, t) \in(0,1) \times(0, T], \\
u(x, t)= & \phi(x, t), x \in[0,1], t \in[-s, 0], \\
u(0, t)= & \gamma(t), u(1, t)=\beta(t), t \in(0, T],
\end{aligned}
$$

where $0<c_{0} \leq D(x) \leq c_{1}$ is the variable diffusion coefficient, $s_{j}>0, j=1,2, \cdots, q$ are delay terms, and $s=\max _{1 \leq i \leq q}\left\{s_{i}\right\}$; time fractional partial derivative $\left(\partial^{\alpha} u / \partial t^{\alpha}\right)(0<\alpha<1)$ is defined as

$$
\frac{\partial^{\alpha} u}{\partial t^{\alpha}}=\frac{1}{\Gamma(1-\alpha)} \int_{0}^{t}(t-\xi)^{-\alpha} \frac{\partial u(x, \xi)}{\partial \xi} d \xi
$$

where $\Gamma(\cdot)$ is the gamma function.
For simplicity, we consider the following equation with arbitrary two different delays:

$$
\begin{aligned}
\frac{\partial^{\alpha} u}{\partial t^{\alpha}}-\left(D(x) u_{x}\right)_{x}= & f\left(u(x, t), u\left(x, t-s_{1}\right), u\left(x, t-s_{2}\right), x, t\right), \\
& (x, t) \in(0,1) \times(0, T], \\
u(x, t)= & \phi(x, t), x \in[0,1], \quad t \in[-s, 0], \\
u(0, t)= & \gamma(t), u(1, t)=\beta(t), t \in(0, T],
\end{aligned}
$$

where $s_{2}>s_{1}>0$ are two delay terms and satisfy $s_{1}=\left(m_{1}+\right.$ $\left.\delta_{1}\right) \tau, s_{2}=\left(m_{2}+\delta_{2}\right) \tau, m_{2}>m_{1}>0$ are integers, and $\delta_{1}, \delta_{2} \epsilon$ $[0,1], s=\left(m_{2}+1\right) \tau$.

Applications of delay differential equations (DDEs) can be found in many fields, such as electrotechnics, population dynamics, biology, and economics [1-5]. However, one can obtain the analytical solutions of DDEs in few cases. The researchers turn to numerical methods for solving DDEs in general cases [6-9]. Most studies about DDEs consider one delay; in fact, more than one delays should be considered in some systems. Tian and Kuang considered $\mathrm{H}$-method and GP-stability for DDEs with several delay terms [10]; the applications of such DDEs can be found in [11-14].

Fractional delay differential equations (FDDEs) are widely used in automatic control, population dynamics, 
finance, etc. [15-17]. Numerical solutions for FDDEs can be found in [18-25], where [18-20] considered numerical solutions for FDDEs, and [21-25] considered numerical solutions for fractional delay partial differential equations (FDPDEs). However, the researchers seldom considered the numerical methods for FDDEs with multidelays; some studies considered such FDDEs without theoretical analysis.

In this paper, there is an effective difference scheme for solving systems (3)-(5) with multidelay. Some stability and convergence results of the scheme are obtained by mathematical proof, and the theoretical results are proved by two numerical tests.

We organize the paper as the follows. Section 2 constructs a numerical scheme to solve (3)-(5). Section 3 provides the stability and convergence results by proof. Section 4 presents two numerical tests. Section 5 gives a brief discussion.

\section{The Construction of the Second Finite Difference Method}

The following assumptions are assumed to be true in this paper.

(H1) Assume $D(x)$ to be sufficiently smooth function which fulfills $0<c_{0} \leq D(x) \leq c_{1}$

(H2) Suppose that $f\left(u(x, t), u\left(x, t-s_{1}\right), u\left(x, t-s_{2}\right), x, t\right)$ is sufficiently smooth and satisfies $[26,27]$

$$
\begin{aligned}
& \left|f\left(\mu+\varepsilon_{1}, \nu+\varepsilon_{2}, \iota+\varepsilon_{3}, x, t\right)-f(\mu, \nu, \iota, x, t)\right| \\
& \quad \leq c_{2}\left|\varepsilon_{1}\right|+c_{3}\left|\varepsilon_{2}\right|+c_{4}\left|\varepsilon_{3}\right|,
\end{aligned}
$$

where $\varepsilon_{1}, \varepsilon_{2}, \varepsilon_{3}$ are arbitrary real numbers and $c_{2}>0, c_{3}>0$, and $c_{4}>0$ are three constants.

Taking $M>0$ and $N>0$ to be two integers, we have space and time stepsize $h=1 / M$ and $\tau=T / N$, respectively, and discrete points $x_{i}=i h$ and $t_{k}=k \tau$. Assume $\Omega_{h \tau}=\Omega_{h} \times \Omega_{\tau}, \Omega_{h}$ $=\left\{x_{i} \mid 0 \leq i \leq M\right\}, \Omega_{\tau}=\left\{t_{k} \mid-\left(m_{2}+1\right) \leq k \leq N\right\}$, and $U_{i}^{k}=u$ $\left(x_{i}, t_{k}\right)$. Define the following grid function space on $\Omega_{h \tau}$ :

$$
\mathscr{W}=\left\{v_{i}^{k} \mid 0 \leq i \leq M,-\left(m_{2}+1\right) \leq k \leq N\right\}[r g b] 1.00,0.00,0.00,
$$

$$
r_{i}^{k}=\frac{1}{\Gamma(2-\alpha)}\left[\frac{1-\alpha}{12}+\frac{2^{2-\alpha}}{2-\alpha}-\left(1+2^{-\alpha}\right)\right] \max _{0 \leq t \leq t_{k}}\left|\frac{\partial^{2} u\left(x_{i}, t_{k}\right)}{\partial t^{2}}\right| \tau^{2-\alpha}
$$

We have

$$
\begin{aligned}
\delta_{x} v_{i+1 / 2}^{k} & =\frac{v_{i+1}^{k}-v_{i}^{k}}{h}, \delta_{x}^{2} v_{i}^{k}=\frac{v_{i+1}^{k}-2 v_{i}^{k}+v_{i-1}^{k}}{h^{2}}, \delta_{x}\left(D \delta_{x} v\right)_{i}^{k} \\
& =\left(D_{i+1 / 2} \delta_{x} v_{i+1 / 2}^{k}-D_{i-1 / 2} \delta_{x} v_{i-1 / 2}^{k}\right) / h, \\
\delta_{t}^{\alpha} v^{k} & =\frac{\tau^{-\alpha}}{\Gamma(2-\alpha)}\left[d_{0} v^{k}-\sum_{j=1}^{k-1}\left(d_{k-j-1}-d_{k-j}\right) v^{j}-d_{k-1} v^{0}\right],
\end{aligned}
$$

where $d_{j}=(j+1)^{1-\alpha}-j^{1-\alpha}, j \geq 0$.
We introduce the following two Lemmas,

Lemma 1 [28]. Suppose $0<\alpha<1, y \in C^{2}\left[0, t_{k}\right]$; it holds that

$$
\begin{aligned}
& \mid \frac{1}{\Gamma(1-\alpha)} \int_{0}^{t_{k}} \frac{y^{\prime}(s) d s}{\left(t_{k}-s\right)^{\alpha}}-\frac{\tau^{-\alpha}}{\Gamma(2-\alpha)} \\
& \cdot\left[d_{0} y\left(t_{k}\right)-\sum_{j=1}^{k-1}\left(d_{k-j-1}-d_{k-j}\right) y\left(t_{j}\right)-d_{k-1} y\left(t_{0}\right)\right] \mid \\
& \quad \leq \frac{1}{\Gamma(2-\alpha)}\left[\frac{1-\alpha}{12}+\frac{2^{2-\alpha}}{2-\alpha}-\left(1+2^{-\alpha}\right)\right] \max _{0 \leq t \leq t_{k}}\left|y^{\prime \prime}(t)\right| \tau^{2-\alpha} .
\end{aligned}
$$

Lemma 2 [29]. Assume $0<\alpha<1$; then, it holds that

(i) $d_{j}$ decrease monotonically with $j$ increases, and $0<$ $d_{j} \leq 1$

(ii) $d_{0}=1, \sum_{j=1}^{k-1}\left(d_{k-j-1}-d_{k-j}\right)=d_{0}-d_{k-1}$

Considering (3) at the point $\left(x_{i}, t_{k}\right)$, we have

From Lemma 1, we obtain

$$
\frac{\partial^{\alpha} u}{\partial t^{\alpha}}\left(x_{i}, t_{k}\right)=\delta_{t}^{\alpha} U_{i}^{k}+r_{i}^{k}
$$

where

From Taylor's expansion, we have

$$
\begin{aligned}
D_{i+1 / 2} \delta_{x} U_{i+1 / 2}^{k}= & D_{i+1 / 2} \frac{\partial u}{\partial x}\left(x_{i+1 / 2}, t_{k}\right) \\
& +\frac{h^{2}}{24} D_{i+1 / 2} \frac{\partial^{3} u}{\partial x^{3}}\left(x_{i+1 / 2}, t_{k}\right)+O\left(h^{3}\right) \\
D_{i-1 / 2} \delta_{x} U_{i-1 / 2}^{k}= & D_{i-1 / 2} \frac{\partial u}{\partial x}\left(x_{i-1 / 2}, t_{k}\right) \\
& +\frac{h^{2}}{24} D_{i-1 / 2} \frac{\partial^{3} u}{\partial x^{3}}\left(x_{i-1 / 2}, t_{k}\right)+O\left(h^{3}\right)
\end{aligned}
$$

$$
\begin{aligned}
& \frac{\partial^{\alpha} u}{\partial t^{\alpha}}\left(x_{i}, t_{k}\right)-\left.\frac{\partial}{\partial x}\left(D(x) \frac{\partial u(x, t)}{\partial x}\right)\right|_{\left(x_{i}, t_{k}\right)} \\
& \quad=f\left(u\left(x_{i}, t_{k}\right), u\left(x_{i}, t_{k}-s_{1}\right), u\left(x_{i}, t_{k}-s_{2}\right), x_{i}, t_{k}\right), 0 \leq i \\
& \quad \leq M, 0 \leq k \leq N .
\end{aligned}
$$



obtain

Subtracting (13) and (14) and dividing the result by $h$, we

$$
\begin{aligned}
\delta_{x}\left(D \delta_{x} U\right)_{i}^{k}= & \frac{1}{h}\left(D_{i+1 / 2} \delta_{x} U_{i+1 / 2}^{k}-D_{i-1 / 2} \delta_{x} U_{i-1 / 2}^{k}\right) \\
= & \frac{1}{h}\left(D_{i+1 / 2} \frac{\partial u}{\partial x}\left(x_{i+1 / 2}, t_{k}\right)-D_{i-1 / 2} \frac{\partial u}{\partial x}\left(x_{i-1 / 2}, t_{k}\right)\right) \\
& +O\left(h^{2}\right)=\left.\frac{\partial}{\partial x}\left(D(x) \frac{\partial u(x, t)}{\partial x}\right)\right|_{\left(x_{i}, t_{k}\right)}+O\left(h^{2}\right)
\end{aligned}
$$

$$
\begin{aligned}
& f\left(u\left(x_{i}, t_{k}\right), u\left(x_{i}, t_{k}-s_{1}\right), u\left(x_{i}, t_{k}-s_{2}\right), x_{i}, t_{k}\right) \\
& \quad=f\left(2 U_{i}^{k-1}-U_{i}^{k-2}, U_{i}^{k-m_{1}-\delta_{1}}, U_{i}^{k-m_{2}-\delta_{2}}, x_{i}, t_{k}\right) \\
& \quad+\tau^{2} \frac{\partial^{2} u}{\partial t^{2}}\left(x_{i}, \eta_{i}^{k}\right) f_{\mu}\left(\rho_{k}, U_{i}^{k-m_{1}-\delta_{1}}, U_{i}^{k-m_{2}-\delta_{2}}, x_{i}, t_{k}\right) .
\end{aligned}
$$

where $U_{i}^{k-m_{1}-\delta_{1}}=\left(1-\delta_{1}\right) U_{i}^{k-m_{1}}+\delta_{1} U_{i}^{k-m_{1}+1}, \quad U_{i}^{k-m_{2}-\delta_{2}}=(1$ $\left.-\delta_{2}\right) U_{i}^{k-m_{2}}+\delta_{2} U_{i}^{k-m_{2}+1}, \eta_{i}^{k} \in\left(t_{k}-s_{2}, t_{k}\right)$, and $\rho_{k}$ is a real number between $u\left(x_{i}, t_{k}\right)$ and $2 U_{i}^{k-1}-U_{i}^{k-2}$.

Substituting (11), (15), and (16) into (10), we obtain

$$
\begin{aligned}
& \delta_{t}^{\alpha} U_{i}^{k}-\delta_{\mathrm{x}}\left(D \delta_{x} U\right)_{i}^{k} \\
& \quad=f\left(2 U_{i}^{k-1}-U_{i}^{k-2}, U_{i}^{k-m_{1}-\delta_{1}}, U_{i}^{k-m_{2}-\delta_{2}}, x_{i}, t_{k}\right)+R_{i}^{k},
\end{aligned}
$$

where

$$
\begin{aligned}
R_{i}^{k}= & r_{i}^{k}+\tau^{2} \frac{\partial^{2} u}{\partial t^{2}}\left(x_{i}, \eta_{i}^{k}\right) f_{\mu}\left(\rho_{k}, U_{i}^{k-m_{1}-\delta_{1}}, U_{i}^{k-m_{2}-\delta_{2}}, x_{i}, t_{k}\right) \\
& +O\left(h^{2}\right) .
\end{aligned}
$$

From Assumptions (H1) and (H2), we can easily obtain

$$
\left|R_{i}^{k}\right| \leq C_{R}\left(\tau^{2-\alpha}+h^{2}\right), \quad 0 \leq i \leq M, 1 \leq k \leq N .
$$

We have the discretization of (4) and (5):

$$
\begin{aligned}
& U_{i}^{k}=\phi\left(x_{i}, t_{k}\right), \quad 0 \leq i \leq M,-\left(m_{2}+1\right) \leq k \leq 0, \\
& U_{0}^{k}=\gamma\left(t_{k}\right), \quad U_{M}^{k}=\beta\left(t_{k}\right), \quad 1 \leq k \leq N .
\end{aligned}
$$

Replacing $U_{i}^{k}$ by $u_{i}^{k}$ in (17), (20), and (21), and omitting $R_{i}^{k}$, we have the following numerical scheme:

$$
\begin{aligned}
& \delta_{t}^{\alpha} u_{i}^{k}-\delta_{x}\left(D \delta_{x} u\right)_{i}^{k} \\
& \quad=f\left(2 u_{i}^{k-1}-u_{i}^{k-2}, u_{i}^{k-m_{1}-\delta_{1}}, u_{i}^{k-m_{2}-\delta_{2}}, x_{i}, t_{k}\right), \\
& u_{i}^{k}=\phi\left(x_{i}, t_{k}\right), \quad 0 \leq i \leq M,-\left(m_{2}+1\right) \leq k \leq 0, \\
& u_{0}^{k}=\gamma\left(t_{k}\right), \quad u_{M}^{k}=\beta\left(t_{k}\right), \quad 1 \leq k \leq N .
\end{aligned}
$$

\section{The Solvability, Convergence, and Stability of the Difference Scheme}

Assume the following to be defined on $\Omega_{h}$

$$
\mathscr{V}_{h, 0}=\left\{u \mid u=\left(u_{0}, u_{1}, \cdots, u_{M}\right), u_{0}=u_{M}=0\right\}
$$

If $u, v \in \mathscr{V}_{h, 0}$, the following inner products and corresponding norms are introduced

$$
\begin{aligned}
(u, v) & =h \sum_{i=1}^{M-1} u_{i} v_{i}, \\
\|u\| & =\sqrt{(u, u)}, \\
\|u\|_{\infty} & =\max _{1 \leq i \leq M-1}\left|u_{i}\right|, \\
\left\langle\delta_{x} u, \delta_{x} v\right\rangle & =h \sum_{i=0}^{M-1}\left(\delta_{x} u_{i+1 / 2}\right)\left(\delta_{x} v_{i+1 / 2}\right), \\
\left|\delta_{x} u\right|_{1} & =\sqrt{\left\langle\delta_{x} u, \delta_{x} u\right\rangle}, \\
\left\langle\delta_{x} u, \delta_{x} v\right\rangle_{D} & =h \sum_{i=0}^{M-1} D\left(x_{i+1 / 2}\right)\left(\delta_{x} u_{i+1 / 2}\right)\left(\delta_{x} v_{i+1 / 2}\right), \\
\left|\delta_{x} u\right|_{1 D} & =\sqrt{\left\langle\delta_{x} u, \delta_{x} u\right\rangle_{D} .}
\end{aligned}
$$

We can obtain Lemma 3 from Assumption (H1):

Lemma 3. For $\forall u \in \mathscr{V}_{h, 0}$, we have $\sqrt{c_{0}}\left|\delta_{x} u\right|_{1} \leq\left|\delta_{x} u\right|_{1 D} \leq$ $\sqrt{c_{1}}\left|\delta_{x} u\right|_{1}$.

Lemma 4 [30]. For $\forall u \in \mathscr{V}_{h, 0}$, one can obtain

$$
\|u\|_{\infty} \leq\left|\delta_{x} u\right|_{1} / 2,\|u\| \leq\left|\delta_{x} u\right|_{1} / \sqrt{6}
$$

We also introduce Lemma 5 to be utilized in the following proof.

Lemma 5 [30]. Assuming the sequence $\left\{F^{k} \geq 0 \mid k \geq 0\right\}$ satisfies

$$
F^{k+1} \leq A+B \tau \sum_{i=1}^{k} F^{l}, \quad k=0,1, \cdots
$$

then

$$
F^{k+1} \leq A \exp (B k \tau), \quad k=0,1,2, \cdots
$$

where $A \geq 0$ and $B \geq 0$ are two constants.

Theorem 6. Under the assumptions of (H1) and (H2), the solution of the difference schemes (22)-(24) is unique. 
Proof. Difference schemes (22)-(24) can be reformed as follows:

When $k=1$, we have

$-\frac{D_{i-1 / 2}}{h^{2}} u_{i-1}^{1}+\left(\frac{1}{\lambda}+\frac{D_{i-1 / 2}+D_{i+1 / 2}}{h^{2}}\right) u_{i}^{1}-\frac{D_{i+1 / 2}}{h^{2}} u_{i+1}^{1}=\frac{1}{\lambda} u_{i}^{0}+f_{i}^{1}$,

When $k \geq 2$, we have

$$
\begin{gathered}
-\frac{D_{i-1 / 2}}{h^{2}} u_{i-1}^{k}+\left(\frac{1}{\lambda}+\frac{D_{i-1 / 2}+D_{i+1 / 2}}{h^{2}}\right) u_{i}^{k}-\frac{D_{i+1 / 2}}{h^{2}} u_{i+1}^{k} \\
=\frac{1}{\lambda}\left[\sum_{j=1}^{k-1}\left(d_{k-j-1}-d_{k-j}\right) u_{i}^{j}+d_{k-1} u_{i}^{0}\right]+f_{i}^{k},
\end{gathered}
$$

where $\lambda=\tau^{\alpha} \Gamma(2-\alpha), f_{i}^{k}=f\left(2 u_{i}^{k-1}-u_{i}^{k-2}, u_{i}^{k-m_{1}-\delta_{1}}, u_{i}^{k-m_{2}-\delta_{2}}\right.$, $\left.x_{i}, t_{k}\right), k=1,2, \cdots$. From (30) and (31), we can see that the schemes (22)-(24) are a linear tridiagonal system with strictly diagonally dominant coefficient matrix. Thus, schemes (22)-(31) have a unique solution.

Denote $e_{i}^{k}=U_{i}^{k}-u_{i}^{k}, 0 \leq i \leq M,-\left(m_{2}+1\right) \leq k \leq N$, subtracting (22)-(24) from (17), (20), and (21), respectively, the following error equations can be obtained:

$$
\begin{aligned}
\delta_{t}^{\alpha} e_{i}^{k}-\delta_{x}\left(D \delta_{x} e\right)_{i}^{k} & =p_{i}^{k}+R_{i}^{k}, \\
e_{i}^{k} & =0, \quad 0 \leq i \leq M,-\left(m_{2}+1\right) \leq k \leq 0, \\
e_{0}^{k} & =0, \quad e_{M}^{k}=0, \quad 1 \leq k \leq N .
\end{aligned}
$$

where $p_{i}^{k}=f\left(2 U_{i}^{k-1}-U_{i}^{k-2}, U_{i}^{k-m_{1}-\delta_{1}}, U_{i}^{k-m_{2}-\delta_{2}}, x_{i}, t_{k}\right)-f(2$ $\left.u_{i}^{k-1}-u_{i}^{k-2}, u_{i}^{k-m_{1}-\delta_{1}}, u_{i}^{k-m_{2}-\delta_{2}}, x_{i}, t_{k}\right)$.

Theorem 7. Denote $u(x, t)$ to be the solution of systems (3)-(5), and (22)-(24) has numerical solution $\left\{u_{i}^{k} \mid 0 \leq i \leq M\right.$, $\left.-\left(m_{2}+1\right) \leq k \leq N\right\}$. Then, we have

$$
\left\|e^{k}\right\|_{\infty} \leq C\left(\tau^{2-\alpha}+h^{2}\right), 1 \leq k \leq N
$$

where $C$ is a positive constant independent of $h$ and $\tau$.

Proof. Multiplying (32) with $h \delta_{t}^{\alpha} e_{i}^{k}$, one can have

$$
\begin{array}{r}
h \sum_{i=1}^{M-1}\left(\delta_{t}^{\alpha} e_{i}^{k}\right)\left(\delta_{t}^{\alpha} e_{i}^{k}\right)-h \sum_{i=1}^{M-1}\left(\delta_{x}\left(D \delta_{x} e\right)_{i}^{k}\right)\left(\delta_{t}^{\alpha} e_{i}^{k}\right) \\
=h \sum_{i=1}^{M-1} p_{i}^{k}\left(\delta_{t}^{\alpha} e_{i}^{k}\right)+h \sum_{i=1}^{M-1}\left(R_{i}^{k}\right)\left(\delta_{t}^{\alpha} e_{i}^{k}\right), \\
1 \leq i \leq M-1,0 \leq k \leq N-1 .
\end{array}
$$

Then each term of (36) will be estimated.

$$
h \sum_{i=1}^{M-1}\left(\delta_{t}^{\alpha} e_{i}^{k}\right)\left(\delta_{t}^{\alpha} e_{i}^{k}\right)=\left\|\delta_{t}^{\alpha} e_{i}^{k}\right\|^{2} .
$$

By the discrete Green formula, we have

$$
\begin{aligned}
& -h \sum_{i=1}^{M-1}\left(\delta_{x}\left(D \delta_{x} e\right)_{i}^{k}\right)\left(\delta_{t}^{\alpha} e_{i}^{k}\right) \\
& =h \sum_{i=0}^{M-1} D_{i+1 / 2}\left(\delta_{x} e_{i+1 / 2}^{k}\right)\left(\delta_{t}^{\alpha} \delta_{x} e_{i+1 / 2}^{k}\right) \\
& =\frac{1}{\lambda} h \sum_{i=0}^{M-1} D_{i+1 / 2}\left(\delta_{x} e_{i+1 / 2}^{k}\right)\left[\delta_{x} e_{i+1 / 2}^{k}\right. \\
& \left.-\sum_{j=1}^{k-1}\left(d_{k-j-1}-d_{k-j}\right) \delta_{x} e_{i+1 / 2}^{j}-d_{k-1} \delta_{x} e_{i+1 / 2}^{0}\right] \\
& =\frac{1}{\lambda}\left\{\left|\delta_{x} e^{k}\right|_{1 D}^{2}-\sum_{j=1}^{k-1}\left(d_{k-j-1}-d_{k-j}\right)\left\langle\delta_{x} e^{k}, \delta_{x} e^{j}\right\rangle_{D}\right. \\
& \left.-d_{k-1}\left\langle\delta_{x} e^{k}, \delta_{x} e^{0}\right\rangle_{D}\right\} \\
& \geq \frac{1}{\lambda}\left\{\left|\delta_{x} e^{k}\right|_{1 D}^{2}-\sum_{j=1}^{k-1}\left(d_{k-j-1}-d_{k-j}\right) \frac{\left|\delta_{x} e^{k}\right|_{1 D}^{2}+\left|\delta_{x} e^{j}\right|_{1 D}^{2}}{2}\right. \\
& \left.-d_{k-1} \frac{\left|\delta_{x} e^{k}\right|_{1 D}^{2}+\left|\delta_{x} e^{0}\right|_{1 D}^{2}}{2}\right\} \\
& =\frac{1}{\lambda}\left\{\frac{\left|\delta_{x} e^{k}\right|_{1 D}^{2}}{2}-\sum_{j=1}^{k-1}\left(d_{k-j-1}-d_{k-j}\right) \frac{\left|\delta_{x} e^{j}\right|_{1 D}^{2}}{2}\right\} .
\end{aligned}
$$

From the Cauchy-Schwarz inequality, we have

$$
\begin{aligned}
h \sum_{i=1}^{M-1}\left(R_{i}^{k}\right)\left(\delta_{t}^{\alpha} e_{i}^{k}\right) & \leq h \sum_{i=1}^{M-1}\left(\frac{\left(R_{i}^{k}\right)^{2}}{2 \varepsilon}+\frac{\varepsilon}{2}\left(\delta_{t}^{\alpha} e_{i}^{k}\right)^{2}\right) \\
& =\frac{1}{2 \varepsilon}\left\|R_{i}^{k}\right\|^{2}+\frac{\varepsilon}{2}\left\|\delta_{t}^{\alpha} e_{i}^{k}\right\|^{2} .
\end{aligned}
$$

From the Cauchy-Schwarz inequality and Assumption (H2), we have

$$
\begin{aligned}
h \sum_{i=1}^{M-1} p_{i}^{k}\left(\delta_{t}^{\alpha} e_{i}^{k}\right) \leq & \frac{\varepsilon}{2}\left\|\delta_{t}^{\alpha} e_{i}^{k}\right\|^{2}+\frac{1}{2 \varepsilon} h \sum_{i=1}^{M-1}\left(p_{i}^{k}\right)^{2} \\
\leq & \frac{\varepsilon}{2}\left\|\delta_{t}^{\alpha} e_{i}^{k}\right\|^{2}+\frac{1}{2 \varepsilon} h \sum_{i=1}^{M-1}\left[c_{3}\left|2 e_{i}^{k-1}-e_{i}^{k-2}\right|\right. \\
& \left.+c_{4}\left|e_{i}^{k-m_{1}-\delta_{1}}\right|+c_{5}\left|e_{i}^{k-m_{2}-\delta_{2}}\right|\right]^{2} \\
\leq & \frac{\varepsilon}{2}\left\|\delta_{t}^{\alpha} e_{i}^{k}\right\|^{2}+\frac{3 c_{3}^{2}}{2 \varepsilon} h \sum_{i=1}^{M-1}\left(2 e_{i}^{k-1}-e_{i}^{k-2}\right)^{2} \\
& +\frac{3 c_{4}^{2}}{2 \varepsilon} h \sum_{i=1}^{M-1}\left(e_{i}^{k-m_{1}-\delta_{1}}\right)^{2} \\
& +\frac{3 c_{5}^{2}}{2 \varepsilon} h \sum_{i=1}^{M-1}\left(e_{i}^{k-m_{2}-\delta_{2}}\right)^{2}
\end{aligned}
$$




$$
\begin{aligned}
\leq & \frac{\varepsilon}{2}\left\|\delta_{t}^{\alpha} e_{i}^{k}\right\|^{2}+\frac{24 c_{3}^{2}}{2 \varepsilon}\left(\left\|e^{k-1}\right\|^{2}+\left\|e^{k-2}\right\|^{2}\right) \\
& +\frac{3 c_{4}^{2}}{2 \varepsilon}\left(\max \left\{\left\|e^{k-m_{1}}\right\|^{2},\left\|e^{k-m_{1}+1}\right\|^{2}\right\}\right) \\
& +\frac{3 c_{5}^{2}}{2 \varepsilon}\left(\max \left\{\left\|e^{k-m_{2}}\right\|^{2},\left\|e^{k-m_{2}+1}\right\|^{2}\right\}\right) .
\end{aligned}
$$

Inserting (37)-(40) into (36), we obtain

$$
\begin{aligned}
\left\|\delta_{t}^{\alpha} e_{i}^{k}\right\|^{2}+\frac{1}{\lambda}\left\{\frac{\left|\delta_{x} e^{k}\right|_{1 D}^{2}}{2}-\sum_{j=1}^{k-1}\left(d_{\mathrm{k}-j-1}-d_{k-j}\right) \frac{\left|\delta_{x} e^{j}\right|_{1 D}^{2}}{2}\right\} \\
\leq \frac{1}{2 \varepsilon}\left\|R_{i}^{k}\right\|^{2}+\frac{\varepsilon}{2}\left\|\delta_{t}^{\alpha} e_{i}^{k}\right\|^{2}+\frac{\varepsilon}{2}\left\|\delta_{t}^{\alpha} e_{i}^{k}\right\|^{2}+\frac{24 c_{3}^{2}}{2 \varepsilon}\left(\left\|e^{k-1}\right\|^{2}+\left\|e^{k-2}\right\|^{2}\right) \\
\quad+\frac{3 c_{4}^{2}}{2 \varepsilon}\left(\max \left\{\left\|e^{k-m_{1}}\right\|^{2},\left\|e^{k-m_{1}+1}\right\|^{2}\right\}\right) \\
+\frac{3 c_{5}^{2}}{2 \varepsilon}\left(\max \left\{\left\|e^{k-m_{2}}\right\|^{2},\left\|e^{k-m_{2}+1}\right\|^{2}\right\}\right) .
\end{aligned}
$$

Multiplying (41) by $2 \lambda$, and letting $\varepsilon=1$, we have

$$
\begin{aligned}
\left|\delta_{x} e^{k}\right|_{1 D}^{2} \leq & \sum_{j=1}^{k-1}\left(d_{k-j-1}-d_{k-j}\right)\left|\delta_{x} e^{j}\right|_{1 D}^{2} \\
& +\lambda\left\|R_{i}^{k}\right\|^{2}+24 \lambda c_{3}^{2}\left(\left\|e^{k-1}\right\|^{2}+\left\|e^{k-2}\right\|^{2}\right) \\
& +3 \lambda c_{4}^{2}\left(\max \left\{\left\|e^{k-m_{1}}\right\|^{2},\left\|e^{k-m_{1}+1}\right\|^{2}\right\}\right) \\
& +3 \lambda c_{5}^{2}\left(\max \left\{\left\|e^{k-m_{2}}\right\|^{2},\left\|e^{k-m_{2}+1}\right\|^{2}\right\}\right) .
\end{aligned}
$$

From Lemmas 3 and 4, and noticing (19), we obtain

$$
\begin{aligned}
\left|\delta_{x} e^{k}\right|_{1}^{2} \leq & \frac{c_{1}}{c_{0}} \sum_{j=1}^{k-1}\left(d_{k-j-1}-d_{k-j}\right)\left|\delta_{x} e^{j}\right|_{1}^{2}+\frac{\lambda}{c_{0}} C_{R}^{2}\left(\tau^{2-\alpha}+h^{2}\right)^{2} \\
& +\frac{4 \lambda c_{3}^{2}}{c_{0}}\left(\left|\delta_{x} e^{k-1}\right|_{1}^{2}+\left|\delta_{x} e^{k-2}\right|_{1}^{2}\right) \\
& +\frac{\lambda c_{4}^{2}}{2 c_{0}}\left(\max \left\{\left|\delta_{x} e^{k-m_{1}}\right|_{1}^{2},\left|\delta_{x} e^{k-m_{1}+1}\right|_{1}^{2}\right\}\right) \\
& +\frac{\lambda c_{5}^{2}}{2 c_{0}}\left(\max \left\{\left|\delta_{x} e^{k-m_{2}}\right|_{1}^{2},\left|\delta_{x} e^{k-m_{2}+1}\right|_{1}^{2}\right\}\right),
\end{aligned}
$$

denoting

$$
C_{k}=\frac{1}{c_{0}} \Gamma(2-\alpha) \max \left\{C_{R}^{2}, 4 c_{3}^{2}, c_{4}^{2} / 2, c_{5}^{2} / 2\right\}>0
$$

Noticing $\lambda=\tau^{\alpha} \Gamma(2-\alpha)$, one can obtain $\tau^{\alpha}<1$ from $0<$ $\tau<1$. By (43), one can have

$$
\begin{aligned}
\left|\delta_{x} e^{k}\right|_{1}^{2} \leq & \frac{c_{1}}{c_{0}} \sum_{j=1}^{k-1}\left(d_{k-j-1}-d_{k-j}\right)\left|\delta_{x} e^{j}\right|_{1}^{2} \\
& +C_{k}\left[\left(\tau^{2-\alpha}+h^{2}\right)^{2}+\left|\delta_{x} e^{k-1}\right|_{1}^{2}+\left|\delta_{x} e^{k-2}\right|_{1}^{2}\right. \\
& +\max \left\{\left|\delta_{x} e^{k-m_{1}}\right|_{1}^{2},\left|\delta_{x} e^{k-m_{1}+1}\right|_{1}^{2}\right\} \\
& \left.+\max \left\{\left|\delta_{x} e^{k-m_{2}}\right|_{1}^{2},\left|\delta_{x} e^{k-m_{2}+1}\right|_{1}^{2}\right\}\right] .
\end{aligned}
$$

From Lemmas 2 and 5, we have

$$
\begin{aligned}
& \left|\delta_{x} e^{k}\right|_{1}^{2} \leq C_{k} \exp \left(4 C_{k}+\frac{c_{1}}{c_{0}} \sum_{j=1}^{k-1}\left(d_{k-j-1}-d_{k-j}\right)\right)\left(\tau^{2-\alpha}+h^{2}\right)^{2} \\
& =C_{k} \exp \left(4 C_{k}+\frac{c_{1}}{c_{0}}\left(1-d_{k-1}\right)\right)\left(\tau^{2-\alpha}+h^{2}\right)^{2} \\
& \leq C_{1}\left(\tau^{2-\alpha}+h^{2}\right)^{2}
\end{aligned}
$$

where $C_{1}=C_{k} \exp \left(4 C_{k}+\left(c_{1} / c_{0}\right)\right)$. From Lemma 4 , we have

$$
\left\|e^{k}\right\|_{\infty} \leq \sqrt{C_{1}\left(\tau^{2-\alpha}+h^{2}\right)^{2}}=C\left(\tau^{2-\alpha}+h^{2}\right) .
$$

We complete the proof.

To discuss the stability of the difference schemes (22)-(24), we consider the following problem:

$$
\frac{\partial^{\alpha} v}{\partial t^{\alpha}}-\left(D(x) v_{x}\right)_{x}=f\left(v(x, t), v\left(x, t-s_{1}\right), v\left(x, t-s_{2}\right), x, t\right),
$$

$$
(x, t) \in(0,1) \times(0, T],
$$

$$
\begin{aligned}
& v(x, t)=\phi(x, t)+\psi(x, t), \quad x \in[0,1], \quad t \in[-s, 0], \\
& v(0, t)=\gamma(t), \quad u(1, t)=\beta(t), \quad t \in(0, T],
\end{aligned}
$$

where $\psi(x, t)$ is the perturbation caused by $\phi(x, t)$. The following difference scheme solving for (48)-(50) can be obtained:

$$
\delta_{t}^{\alpha} u_{i}^{k}-\delta_{x}\left(D \delta_{x} u\right)_{i}^{k}=f\left(2 u_{i}^{k-1}-u_{i}^{k-2}, u_{i}^{k-m_{1}-\delta_{1}}, u_{i}^{k-m_{2}-\delta_{2}}, x_{i}, t_{k}\right),
$$

$$
\begin{aligned}
& u_{i}^{k}=\phi\left(x_{i}, t_{k}\right)+\psi_{i}^{k}, \quad 0 \leq i \leq M,-\left(m_{2}+1\right) \leq k \leq 0, \\
& u_{0}^{k}=\gamma\left(t_{k}\right), \quad u_{M}^{k}=\beta\left(t_{k}\right), \quad 1 \leq k \leq N .
\end{aligned}
$$


TABLE 1: Simulation results of Example 10, where $h=1 / 2000$ and $\alpha=0.4,0.6,0.8$.

\begin{tabular}{lcccccc}
\hline$\tau$ & $\begin{array}{c}\alpha=0.4 \\
e(h, \tau)\end{array}$ & Rate $_{\tau}$ & $\begin{array}{c}\alpha=0.6 \\
e(h, \tau)\end{array}$ & Rate $_{\tau}$ & $\begin{array}{c}\alpha=0.8 \\
e(h, \tau)\end{array}$ & Rat $_{\mathrm{e}_{\tau}}$ \\
\hline $1 / 50$ & $4.244 \mathrm{e}-005$ & $*$ & $1.752 \mathrm{e}-004$ & $*$ & $6.464 \mathrm{e}-004$ & $*$ \\
$1 / 100$ & $1.609 \mathrm{e}-005$ & 1.399 & $6.903 \mathrm{e}-005$ & 1.344 & $2.851 \mathrm{e}-004$ & 1.181 \\
$1 / 150$ & $8.950 \mathrm{e}-006$ & 1.446 & $3.963 \mathrm{e}-005$ & 1.369 & $1.758 \mathrm{e}-004$ & 1.192 \\
$1 / 200$ & $6.401 \mathrm{e}-006$ & 1.165 & $2.724 \mathrm{e}-005$ & 1.303 & $1.253 \mathrm{e}-004$ & 1.178 \\
\hline
\end{tabular}

Denote

$$
\omega_{i}^{k}=v_{i}^{k}-u_{i}^{k}, \quad 0 \leq i \leq M,-\left(m_{2}+1\right) \leq k \leq N .
$$

where $\bar{C}$ is a bounded constant independent of $h$ and $\tau$.

Definition 8. Assume $u_{i}^{k}$ satisfy (22)-(24) and $v_{i}^{k}$ satisfy (51)-(53), a numerical scheme for (3)-(5) is stable if we have

$$
\left\|\omega^{k}\right\|_{\infty} \leq \bar{C} \max _{-\left(m_{2}+1\right) \leq j \leq 0}\left|\psi^{j}\right|_{1}
$$

Similar to the proof of Theorem 7, the following stability result can be obtained.

Theorem 9. Under the same condition as Theorem 7, numerical schemes (22)-(24) satisfy $\left\|\omega^{k}\right\|_{\infty} \leq \widehat{C} \max _{-\left(m_{2}+1\right) \leq j \leq 0}\left|\psi^{j}\right|_{1}$.

\section{Numerical Test}

Now, we present the following numerical tests to testify the schemes (22)-(24). Introducing the following notations,

$$
\begin{aligned}
e(h, \tau) & =\max _{1 \leq k \leq N}\left\|U^{k}-u^{k}\right\|_{\infty}, \\
\text { Rate }_{\tau} & =\frac{\log \left(e\left(h, \tau_{1}\right) / e\left(h, \tau_{2}\right)\right)}{\log \left(\tau_{1} / \tau_{2}\right)}, \\
\text { Rate }_{h} & =\frac{\log \left(e\left(h_{1}, \tau\right) / e\left(h_{2}, \tau\right)\right)}{\log \left(h_{1} / h_{2}\right)} .
\end{aligned}
$$

$$
\left\{\begin{array}{l}
\frac{\partial^{\alpha} u}{\partial t^{\alpha}}-\frac{\partial u}{\partial x}\left(x^{2} \frac{\partial u}{\partial x}\right)=-u(x, t)^{2}+u(x, t-0.1)+u(x, t-0.2)+G(x, t),(x, t) \in(0,1) \times(0, T], \\
u(x, t)=e^{x} x^{2}(1-x)^{2} t^{2+\alpha}, \quad x \in[0,1], \quad t \in[-0.2,0] \\
u(0, t)=0, \quad u(1, t)=0, \quad t \in(0,1] .
\end{array} .\right.
$$

TABLE 2: Simulation results of Example 10, where $\tau=1 / 2000$ and $\alpha=0.4,0.6,0.8$.

\begin{tabular}{lcccccc}
\hline$h$ & $\begin{array}{c}\alpha=0.4 \\
e(h, \tau)\end{array}$ & Rate $_{h}$ & $\begin{array}{c}\alpha=0.6 \\
e(h, \tau)\end{array}$ & Rate $_{h}$ & $\begin{array}{c}\alpha=0.8 \\
e(h, \tau)\end{array}$ & Rate $_{h}$ \\
\hline $1 / 10$ & $6.213 \mathrm{e}-002$ & $*$ & $6.072 \mathrm{e}-002$ & $*$ & $5.889 \mathrm{e}-002$ & $*$ \\
$1 / 20$ & $1.534 \mathrm{e}-002$ & 2.018 & $1.500 \mathrm{e}-002$ & 2.017 & $1.455 \mathrm{e}-002$ & 2.017 \\
$1 / 40$ & $3.842 \mathrm{e}-003$ & 1.997 & $3.758 \mathrm{e}-003$ & 1.997 & $3.654 \mathrm{e}-003$ & 1.994 \\
$1 / 80$ & $9.599 \mathrm{e}-004$ & 2.001 & $9.396 \mathrm{e}-004$ & 2.000 & $9.185 \mathrm{e}-004$ & 1.992 \\
\hline
\end{tabular}

when considering for Rate ${ }_{\tau}, h$ should be fixed and small enough. While considering for Rate ${ }_{h}, \tau$ should be fixed and small enough.

$$
\left\{\begin{array}{l}
\frac{\partial^{\alpha} u}{\partial t^{\alpha}}-\frac{\partial u}{\partial x}\left(\left(x^{2}+1\right) \frac{\partial u}{\partial x}\right)=\frac{u(x, t-0.1)}{1+u^{2}(x, t-0.05)}+G(x, t), \\
(x, t) \in(0,1) \times(0, T], \\
u(x, t)=t^{2+\alpha} \cos (2 \pi x), \quad x \in[0,1], \quad t \in[-0.1,0], \\
u(0, t)=t^{2+\alpha}, \quad u(1, t)=t^{2+\alpha}, \quad t \in(0,1] .
\end{array}\right.
$$

Example 10. Let $D(x)=x^{2}+1$.

Equation (57) has exact solution $u(x, t)=t^{2+\alpha} \cos (2 \pi x)$, where

$$
\begin{aligned}
G(x, t)= & {\left[\frac{\Gamma(3+\alpha)}{\Gamma(3)} t^{2}+4 \pi^{2}\left(x^{2}+1\right) t^{2+\alpha}\right] \cos (2 \pi x) } \\
& +4 \pi x t^{2+\alpha} \sin (2 \pi x) \\
& -\frac{(t-0.1)^{2+\alpha} \cos (2 \pi x)}{1+(t-0.05)^{4+2 \alpha} \cos ^{2}(2 \pi x)}
\end{aligned}
$$

Table 1 presents the maximum errors in the temporal directions, where $\alpha=0.4,0.6,0.8$, and $h=1 / 2000$. From the results, we can draw a conclusion that the convergence order in the temporal directions is $2-\alpha$. Table 2 presents the maximum errors in the spatial directions, where $\alpha=0.4,0.6,0.8$, and $\tau=1 / 2000$. From the results, we can draw a conclusion that the convergence order in the spatial directions is 2 .

Figure 1 gives the error planes of Example 10, where $\tau=h=1 / 100$, and $\alpha=0.1,0.3,0.7,0.9$. Figure 1 tells us that bigger $\alpha$ can bring bigger error.

Example 11. Let $D(x)=x^{2}+1$ 

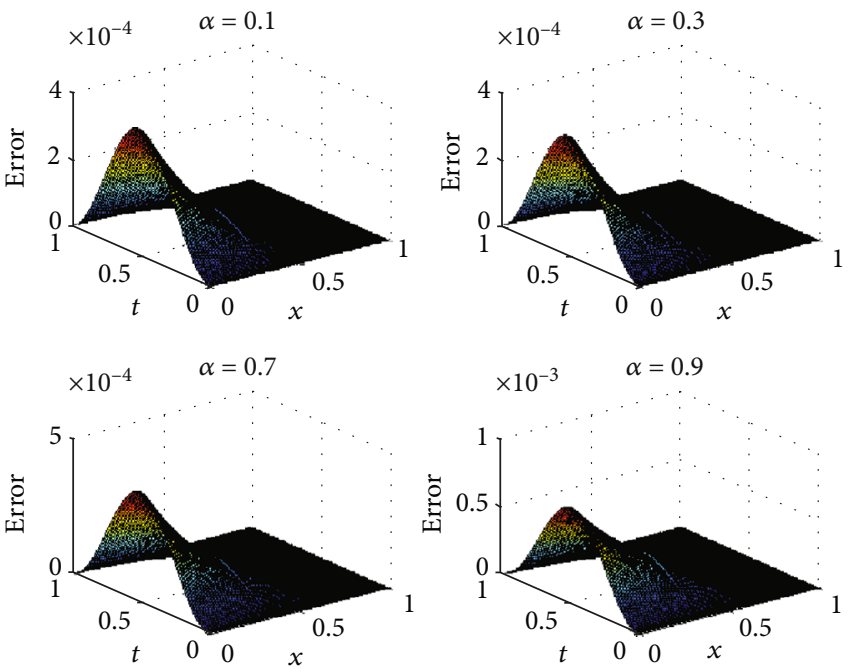

FIgURE 1: Error planes of Example 10, where $\tau=h=1 / 100$.

TABLE 3: Simulation results of Example 11, where $h=1 / 2000$ and $\alpha=0.3,0.5,0.8$.

\begin{tabular}{lcccccc}
\hline$\tau$ & $\begin{array}{c}\alpha=0.3 \\
e(h, \tau)\end{array}$ & Rate $_{\tau}$ & $\begin{array}{c}\alpha=0.5 \\
e(h, \tau)\end{array}$ & Rate $_{\tau}$ & $\begin{array}{c}\alpha=0.8 \\
e(h, \tau)\end{array}$ & Rate $_{\tau}$ \\
\hline $1 / 10$ & $9.029 \mathrm{e}-005$ & $*$ & $2.277 \mathrm{e}-004$ & $*$ & $9.049 \mathrm{e}-004$ & $*$ \\
$1 / 20$ & $2.712 \mathrm{e}-005$ & 1.735 & $7.924 \mathrm{e}-005$ & 1.523 & $3.941 \mathrm{e}-004$ & 1.199 \\
$1 / 40$ & $8.183 \mathrm{e}-006$ & 1.729 & $2.763 \mathrm{e}-005$ & 1.520 & $1.713 \mathrm{e}-004$ & 1.202 \\
$1 / 80$ & $2.530 \mathrm{e}-006$ & 1.693 & $9.707 \mathrm{e}-006$ & 1.509 & $7.451 \mathrm{e}-005$ & 1.201 \\
\hline
\end{tabular}

TABLE 4: Simulation results of Example 11, where $\tau=1 / 2000$ and $\alpha=0.3,0.5,0.8$.

\begin{tabular}{lcccccc}
\hline$h$ & $\begin{array}{c}\alpha=0.3 \\
e(h, \tau)\end{array}$ & Rate $_{h}$ & $\begin{array}{c}\alpha=0.5 \\
e(h, \tau)\end{array}$ & Rate $_{h}$ & $\begin{array}{c}\alpha=0.8 \\
e(h, \tau)\end{array}$ & Rate $_{h}$ \\
\hline $1 / 8$ & $8.064 \mathrm{e}-003$ & $*$ & $7.917 \mathrm{e}-003$ & $*$ & $7.624 \mathrm{e}-003$ & $*$ \\
$1 / 16$ & $2.100 \mathrm{e}-003$ & 1.941 & $2.057 \mathrm{e}-003$ & 1.945 & $1.971 \mathrm{e}-003$ & 1.952 \\
$1 / 32$ & $5.272 \mathrm{e}-004$ & 1.994 & $5.163 \mathrm{e}-004$ & 1.994 & $4.957 \mathrm{e}-004$ & 1.991 \\
$1 / 64$ & $1.319 \mathrm{e}-004$ & 1.998 & $1.293 \mathrm{e}-004$ & 1.998 & $1.250 \mathrm{e}-004$ & 1.987 \\
\hline
\end{tabular}

Equation (59) has exact solution $u(x, t)=e^{x} x^{2}(1-x)^{2}$ $t^{2+\alpha}$, where

$$
\begin{aligned}
G(x, t)= & e^{2 x} x^{4}(1-x)^{4} t^{4+2 \alpha}-e^{x} x^{2}(1-x)^{2}(t-0.1)^{2+\alpha} \\
& -e^{x} x^{2}(1-x)^{2}(t-0.2)^{2+\alpha} \\
& +\frac{\Gamma(3+\alpha)}{2} t^{2} e^{x} x^{2}(1-x)^{2} \\
& -e^{x} t^{2+\alpha}\left(6 x^{2}-18 x^{3}+5 x^{4}+8 x^{5}+x^{6}\right) .
\end{aligned}
$$

Tables 3 and 4 show the computational results for a different $\alpha$ of Example 11. When $h=1 / 2000$, the maximum errors and convergence orders are provided in Table 3 . It can be shown that the $2-\alpha$ order accuracy in temporal direction is verified, while when $\tau=1 / 2000$, from Table 4 , we can see that the 2 order accuracy in spatial direction is verified.

\section{Conclusion}

This paper provides a finite difference scheme for solving a type of variable coefficient time fractional subdiffusion equation with multidelay. The unconditional stability and the global convergence of the scheme in the maximum norm are proved. Numerical experiments were provided to support the theoretical results and testify the efficiency of the difference scheme.

\section{Data Availability}

The author declares that the readers can access the data supporting the conclusions of the study online directly.

\section{Conflicts of Interest}

The author declares that there is no conflicts of interests regarding the publication of this article.

\section{Acknowledgments}

This work is supported by the National Natural Science Foundation of China (No. 71974204, 11401591) and supported by the Fundamental Research Funds for the Central Universities (No. 2722020PY042).

\section{References}

[1] R. D. Driver, Ordinary and Delay Differential Equations, Springer-Verlag, New York, 1977.

[2] K. Yang and L. Chrlotte, Delay Differential Equations: With Applications in Population Dynamics, Academic Press, New York, 1993.

[3] J. Wu, Theory and Applications of Partial Functional Differential Equations, Springer, New York, 1996.

[4] J. E. Forde, Delay Differential Equation Models in Mathematical Biology, University of Michigan, Ann Arbor, MI, 2005.

[5] Q. Huang, D. Qiumei, and J. Z. Li, "Numerical investigations of a class of biological models on unbounded domain," 
Numerical Mathematics: Theory, Methods and Applications, vol. 12, no. 1, pp. 169-186, 2019.

[6] D. Li and C. Zhang, "Nonlinear stability of discontinuous Galerkin methods for delay differential equations," Applied Mathematics Letters, vol. 23, no. 4, pp. 457-461, 2010.

[7] D. Li and C. Zhang, "Superconvergence of a discontinuous Galerkin method for first-order linear delay differential equations," Journal of Computational Mathematics, vol. 29, no. 5, pp. 574-588, 2011.

[8] D. Li and C. Zhang, "error estimates of discontinuous Galerkin methods for delay differential equations," Applied Numerical Mathematics, vol. 82, pp. 1-10, 2014.

[9] H. Qin, Q. Zhang, and S. Wan, "The continuous Galerkin finite element methods for linear neutral delay differential equations," Applied Mathematics and Computation, vol. 346, pp. 76-85, 2019.

[10] H. J. Tian and J. X. Kuang, "The stability of the H-methods in the numerical solution of delay differential equations with several delay terms," Journal of Computational and Applied Mathematics, vol. 58, pp. 171-181, 1995.

[11] C. T. Baker, G. A. Bocharov, and A. Rihan, "A report on the use of delay differential equations in numerical modeling in the biosciences," MCCM Technical Report, Manchester, vol. 343, pp. 2-6, 1999.

[12] M. Z. Liu and M. N. Spiker, "The stability of the H-methods in the numerical solution of delay differential equations," IMA Journal of Numerical Analysis, vol. 10, no. 1, pp. 31-48, 1990.

[13] J. X. Kuang, "The PL-stability of block H-methods," Mathematica Numerica Sinica-Chinese Edition, vol. 2, pp. 135-140, 1997.

[14] Q. Zhang, C. Zhang, and L. Wang, "The compact and CrankNicolson ADI schemes for two-dimensional semilinear multidelay parabolic equations," Journal of Computational and Applied Mathematics, vol. 306, pp. 217-230, 2016.

[15] C. Hwang and Y. C. Cheng, "A numerical algorithm for stability testing of fractional delay systems," Automatica, vol. 42, no. 5, pp. 825-831, 2006.

[16] L. C. Davis, "Modification of the optimal velocity traffic model to include delay due to driver reaction time," Physica A, vol. 319, pp. 557-567, 2002.

[17] Y. Kuang, Delay Differential Equations with Applications in Population Biology, Academic Press, Boston (MA), 1993.

[18] S. Bhalekar and V. Daftardar-Gejji, "A predictor-corrector scheme for solving nonlinear delay differential equations of fractional order," Journal of Fractional Calculus and Applications, vol. 1, pp. 1-8, 2011.

[19] V. Daftardar-Gejji and Y. Sukale, "Solving fractional delay differential equations: a new approach," Fractional Calculus and Applied Analysis, vol. 18, pp. 400-418, 2015.

[20] Z. Wang, X. Huang, and J. Zhou, "A numerical method for delayed fractional-order differential equations: based on G-L definition," Applied Mathematics \& Information Sciences, vol. 7, no. 2L, pp. 525-529, 2013.

[21] F. A. Rihan, "Computational methods for delay parabolic and time-fractional partial differential equations," Numerical Methods for Partial Differential Equations, vol. 26, no. 6, pp. 1556-1571, 2010.

[22] M. Sakara, F. Uludag, and F. Erdogan, "Numerical solution of time-fractional nonlinear PDEs with proportional delays by homotopy perturbation method," Applied Mathematical Modelling, vol. 40, no. 13-14, pp. 6639-6649, 2016.
[23] Q. F. Zhang, M. H. Ran, and D. H. Xu, "Analysis of the compact difference scheme for the semilinear fractional partial differential equation with time delay," Applicable Analysis, vol. 96, pp. 1-18, 2016.

[24] Q. Zhang and T. Li, "Asymptotic stability of compact and linear $\$ \$ \backslash$ theta $\$ \$ \theta$-Methods for space fractional delay generalized diffusion equation," Journal of Scientific Computing, vol. 81, no. 3, pp. 2413-2446, 2019.

[25] Q. Zhang, L. Liu, and C. Zhang, "Compact scheme for fractional diffusion-wave equation with spatial variable coefficient and delays," Applicable Analysis, vol. 2020, pp. 1-22, 2020.

[26] J. Xie, D. Deng, and H. Zheng, “A compact difference scheme for one-dimensional nonlinear delay reaction-diffusion equations with variable coefficient," International Journal of Applied Mathematics, vol. 47, no. 1, pp. 14-19, 2017.

[27] F. Wu, X. Cheng, D. Li, and J. Duan, "A two-level linearized compact ADI scheme for two-dimensional nonlinear reaction-diffusion equations," Computers \& Mathematics with Applications, vol. 75, no. 8, pp. 2835-2850, 2018.

[28] Z. Sun and X. Wu, "A fully discrete difference scheme for a diffusion-wave system," Applied Numerical Mathematics, vol. 56, no. 2, pp. 193-209, 2006.

[29] S. Chen, F. Liu, P. Zhuang, and V. Anh, "Finite difference approximations for the fractional Fokker-Planck equation," Applied Mathematical Modelling, vol. 33, no. 1, pp. 256-273, 2009.

[30] Z. Z. Sun, The Numerical Methods for Partial Equations, Science Press, Beijing, 2005, (in Chinese). 